\title{
Anatomic landmark approach to reconstruction of asymmetric midline cleft lip due to Pai syndrome
}

\author{
Danielle L. Sobol ${ }^{1,2}$, Benjamin B. Massenburg ${ }^{1,2}$, Raymond W. Tse ${ }^{1,2}$ \\ ${ }^{1}$ Division of Craniofacial and Plastic Surgery, Department of Surgery, Seattle Children's Hospital, Seattle, WA; ${ }^{2}$ Division of Plastic Surgery, \\ Department of Surgery, University of Washington, Seattle, WA, USA
}

Midline clefts of the upper lip are rare, and it is therefore important that surgeons have access to a methodical approach for when these presentations are encountered. We adapted principles of the anatomic subunit approximation for unilateral cleft lip, to the repair of midline clefts. The overt use of anatomic landmarks to define the repair results in a design that inherently adjusts to varying degrees of clefts and can accommodate asymmetries. The "measure twice, cut once" style is an advantage to new surgeons and to surgeons who seldom encounter this presentation. We describe the details of surgical repair in the context of a patient with Pai syndrome and associated nasal hamartomas that resulted in nasolabial asymmetry. This is the first report of surgical outcome following treatment of Pai syndrome and includes early and 5 -year follow-up. The system of repair that we describe is applicable to both symmetric and asymmetric midline clefts.

Keywords Cleft lip / Surgery / Classification / Reconstructive surgical procedures

Received: May 16, $2020 \bullet$ Revised: July 9, $2020 \bullet$ Accepted: July 10, 2020

pISSN: 2234-6163 • elSSN: 2234-6171 • https://doi.org/10.5999/aps.2020.00864• Arch Plast Surg 2020;47:483-486
Correspondence: Raymond W. Tse Division of Craniofacial and Plastic Surgery, Department of Surgery, Seattle Children's Hospital, 4800 Sand Point Way NE, M/S OB.9.527 Seattle, WA 98105, USA

Tel: +1-206-9877488

Fax: +1-206-9873064

E-mail:

raymond.tse@seattlechildrens.org

\section{INTRODUCTION}

Midline cleft of the upper lip, also known as median or Tessier 0 cleft [1], is a rare congenital anomaly that is thought to arise from lack of embryologic fusion of the medial nasal prominences. Cleft surgeons will rarely encounter cases during a career and thus a reliable approach to treatment is important. Although methods of repair have been described [2-6], specific technical details are lacking.

Pai syndrome is characterized by midline cleft lip with the addition of corpus callosum lipomas and facial polyps, that can make the nasolabial region asymmetric $[2,7,8]$. Although this presentation has been relatively recently reported, methods to accommodate for the asymmetry have not been described and treatment outcomes are lacking.
We adapted principles of the anatomic subunit approximation for unilateral cleft lip repair [9] to the repair of midline clefts. Given that the design is based upon anatomic landmarks, it can adapt to variations in presentation, including the asymmetry from Pai syndrome (Fig. 1). We present anatomic details and design, as well as long-term outcome for a patient with Pai syndrome.

\section{IDEA}

Landmarks are identified (Fig. 2) starting with the chelion (ch) and peak of Cupid's bow (cphi). cphi is defined by the convexity of vermilion and location of philtral ridges. Similar to Noordhoff point [10], the location where the red line and vermilion border start to converge and the white roll flattens, defines the point of 


\section{Fig. 1. Clinical case}

A 3-week-old girl with Pai syndrome at initial presentation with asymmetric midline cleft lip and nose.

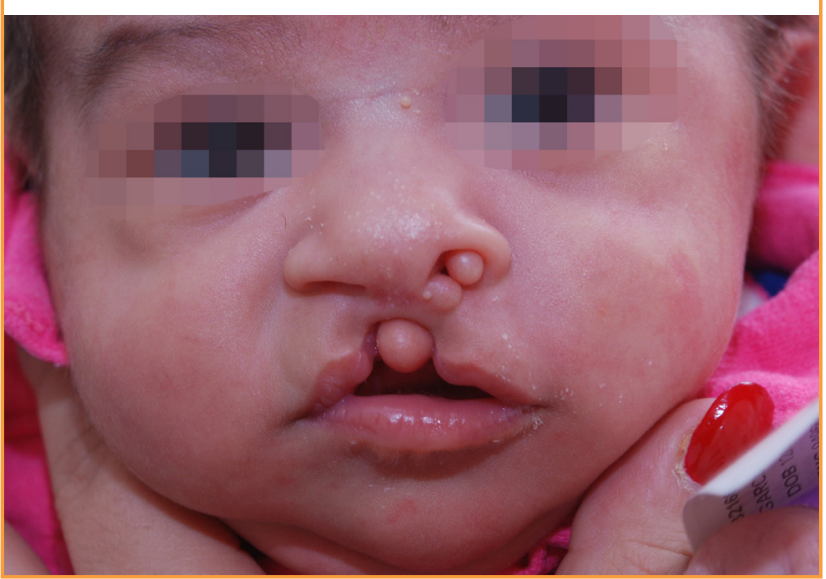

\section{Fig. 2. Anthropometric landmarks and anatomic subunits}

Similar to Noordhoff point, labialis superioris (Is) is defined at the point where the vermilion-mucosal border (red line) sharply converges with the vermilion border and the white roll (white line) begins to flatten. Corresponding landmarks above the white roll (Is$\mathrm{wr}$ ) and along the red line (stomion, sto) are defined. The incision across the vermilion is made along an obtuse curve in order to create a tubercle. Nose landmarks include subalare (sbal), alar insertion point (ai), and superior alar facial groove (safg). The anticipated subnasale (sn) is identified along the crease of the medial footplate (yellow dotted line). Tailoring of hamartoma excision is tailored so that nasal landmarks on either side correspond in position and orientation. The lip is lengthened via the Rose-Thompson effect. ch, chelion; cphi, christa philtri inferioris; cphi-wr, cphi-white roll; cphs, christa philtri superioris.

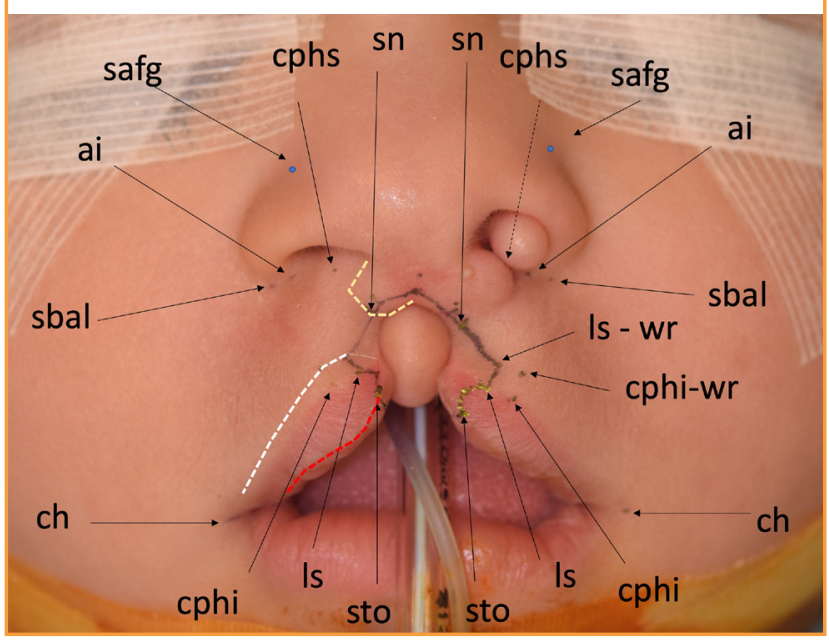

lip closure. Labialis superioris (ls) is marked along the vermilion border and corresponding landmarks above the white roll (lswr) and along the red line (stomion, sto) are defined. Symmetry is verified by measuring corresponding dimensions (ch-cphi, cphi-ls, and ch-sto).

Subalare (sbal) is marked at the lowest point along the ala and

\section{Fig. 3. Intraoral incisions}

Surface landmarks extend to the base of the bifid frenulum ( $\mathrm{fr}$ ). An upper buccal sulcus incision provides access for supraperiosteal dissection along the piriform apertures so that the alar bases and lip can be mobilized. Is, labialis superioris; sn, subnasale; sto, stomion.

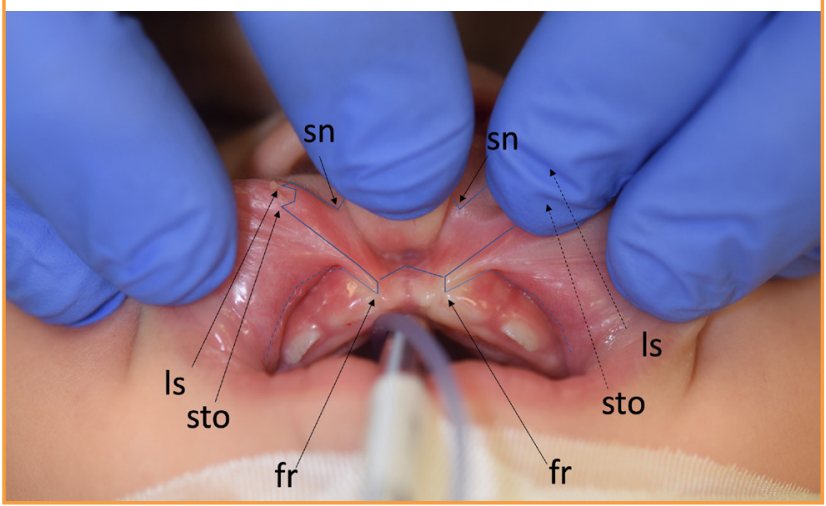

the alar insertion (ai) is marked where the nostril rim meets the nasal sill. The crease that defines the transition between columella and lip is identified so that the subnasale (sn) can be identified on either side of the cleft. The lip-columellar crease normally continues into the nose along the medial footplates.

Incisions are designed perpendicular to the lip so that corresponding lip landmarks (ls-wr, ls, sto) can be approximated (Fig. 2 ). The incision across the vermilion is made with a convex curve in order to produce a midline tubercle upon closure. Further cephalic, the cutaneous lip incisions extend to the cleft apex so that the midline lip can be lengthened via the Rose-Thompson effect [9].

Incisions are extended intraorally along the frenulum and to the alveolus (Fig. 3) so that tissues along and between the cleft margins can be excised. The orbicularis muscle is then released from the alveolus and along the upper buccal sulcus so that it can be mobilized. Further supra-periosteal dissection along the piriform margins mobilizes the alar bases.

Mucosa is then approximated along the midline. The muscle is repaired directly so that the superficial and deep muscle layers can be approximated and the "J" shape of the pars marginalis can be preserved.

Following final cutaneous closure, alar transfixion sutures are placed to contour the nose.

\section{Clinical case}

Reporting of cleft outcome was approved by our institutional review board and the family consented to publication of patient photographs.

A 3-week-old girl with Pai syndrome presented with a midline cleft that extended into the columella and partially into the alveolus (Fig. 1). She had hamartomas of the lip, left sill/footplate, 


\section{Fig. 4. Nasal excisions}

With the lip and columellar elements approximated, the final tailoring is set so that nasal base landmarks are symmetric in position and orientation.

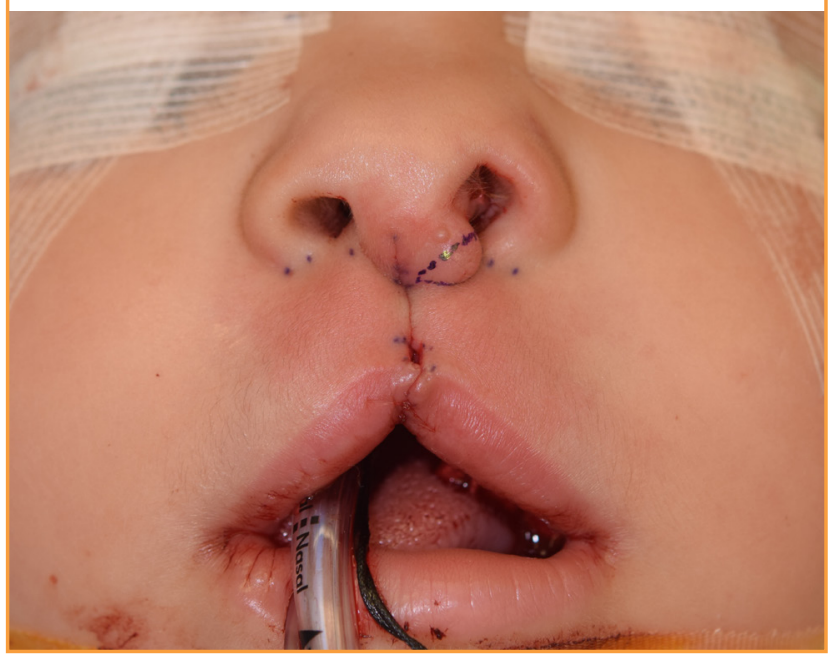

Fig. 5. Postoperative photograph

One year following repair with correction of the nasal base elements and a well-defined tubercle.

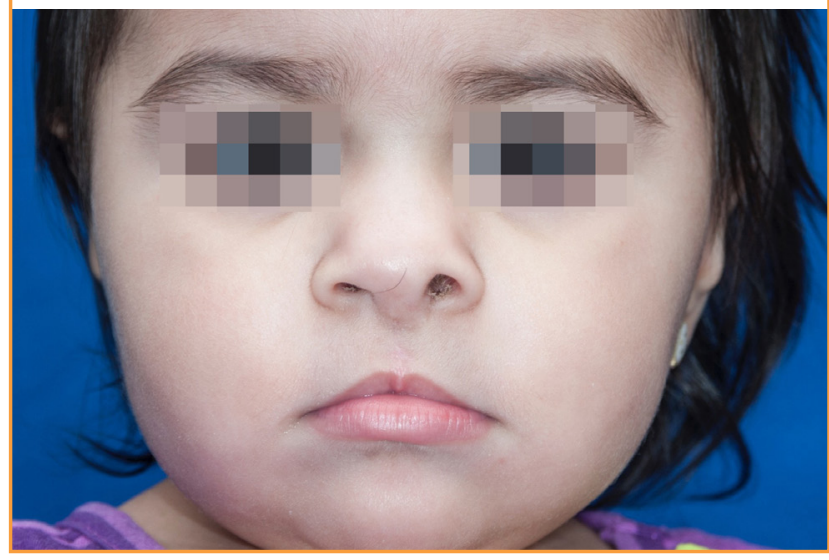

and left septum; and a midline lipoma of corpus callosum. Nasolabial repair was undertaken at 8 months of age.

Her repair followed our proposed approach, however, given the distortion by the hamartomas on the left, the crease on the right (Fig. 2, yellow dotted line) was used as a reference for the left.

With the lip corrected and alar bases mobilized, the cutaneous polyps were excised so that the configuration of nasal base landmarks and nasolabial creases (alar-lip and columellar-lip) matched on either side of the cleft (Fig. 4).

Although a deficiency of the left soft triangle was noted, we deferred correction to a later stage and therefore intentionally left some mild columellar excess.

At 1-year postoperative (Fig. 5), a midline lip tubercle was evident, and correction of nasal sill and nasal base elements re-

\section{Fig. 6. Follow-up at 5 years old}

$(A, B)$ The nasal airway is widely patent. Although the deficiency of soft triangle remains, the family is satisfied with her appearance and will await maturity for final definitive septorhinoplasty.
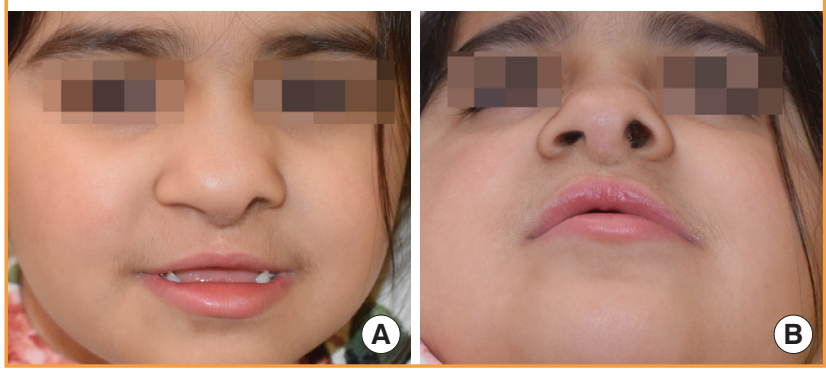

mained symmetric. At 5 years of age (Fig. 6) the family was satisfied with form and function and elected to defer further nasal correction to septorhinoplasty at skeletal maturity.

\section{DISCUSSION}

Midline clefts are estimated to occur in one per one million births [11]. Given how seldom a cleft surgeon will encounter this presentation, an organized and reliable approach can potentially mitigate limited surgical experience. We present a detailed anatomic approach that follows classic reconstructive principles [12] and a measured method of cleft lip repair [9].

Fisher's anatomic subunit approximation for unilateral cleft lip repair [9] was inspired by the concept of anatomic subunits that was proposed by Gonzalez-Ulloa et al. [13] in 1954 and emphasized by Burget and Menick [14] in 1985. The design of the repair [9] considers each anatomic subunit and attempts to place the lines of closure along the borders of subunits. One feature that differentiates it from other methods is the use of anatomic landmarks to define the design for repair [15]. As a result, the approach inherently adjusts to the spectrum of clefts ranging from wide complete to microform clefts. In the case of a midline cleft, we do not believe that all scars can be placed outside of each anatomic subunit, however, we feel that the consideration of each subunit and a system that involves the overt use of landmarks can help to produce favorable form. The columella, medial footplates, sill, philtrum, white roll, vermilion, and mucosa are unique subunits that should be addressed individually. We propose an approach to midline cleft lip repair and outline the anatomic details.

What is critical to this approach is a surgeon's ability to recognize subunits. Although the design may seem involved, the repair can be simplified because each point is a standard anthropometric landmark that experienced surgeons likely already recognize, and new surgeons should learn. Given that we assess 
surgical outcomes using anthropometric analysis, it makes sense that the repair should be based upon those same landmarks. While each surgeon should use the method that they are most comfortable with, the approach that we propose involves a "measure twice, cut once" style that has been associated with a reduced "learning curve" [15]. Those characteristics can be helpful given the rare nature of these clefts and the potential for asymmetric presentations.

Although the foundation of lip and nose for our patient have been addressed, she will eventually benefit from final correction of her deficient soft triangle. We plan to transpose excessive columella tissue or to utilize a composite graft for reconstruction, either when she is motivated for correction or at maturity.

In addition to describing our approach, as far as we know, this is the first report of outcome following surgical treatment of Pai syndrome. The use of anatomic landmarks and subunits to guide primary surgery can help to set the foundation for later stages in care.

\section{NOTES}

\section{Conflict of interest}

No potential conflict of interest relevant to this article was reported.

\section{Ethical approval}

The study was approved by the Institutional Review Board of Seattle Children's Hospital (IRB No. PIROSTUDY14325) and performed in accordance with the principles of the Declaration of Helsinki. Written informed consent was obtained.

\section{Patient consent}

The patient's parent provided written informed consent for the publication and the use of her images.

\section{Author contribution}

Conceptualization: RW Tse. Project administration: RW Tse. Writing - original draft: DL Sobol, BB Massenburg, RW Tse. Writing - review \& editing: DL Sobol, BB Massenburg, RW Tse.

\section{ORCID}

Danielle L. Sobol https://orcid.org/0000-0002-1986-6387 Benjamin B. Massenburg https://orcid.org/0000-0002-8570-5178

Raymond W. Tse https://orcid.org/0000-0002-0179-9809

\section{REFERENCES}

1. Tessier P. Anatomical classification facial, cranio-facial and latero-facial clefts. J Maxillofac Surg 1976;4:69-92.

2. Pai GS, Levkoff AH, Leithiser RE Jr. Median cleft of the upper lip associated with lipomas of the central nervous system and cutaneous polyps. Am J Med Genet 1987;26:921-4.

3. Millard DRJr, Williams S. Median lip clefts of the upper lip. Plast Reconstr Surg 1968;42:4-14.

4. Koh KS, Kim DY, Oh TS. Clinical features and management of a median cleft lip. Arch Plast Surg 2016;43:242-7.

5. Imai Y, Kure S, Nara C, et al. Mental development and surgical prognosis of Pai syndrome: a case report and review of the literature. Cleft Palate Craniofac J 2019;56:273-9.

6. Da Silva Freitas R, Alonso N, Shin JH, et al. Surgical correction of Tessier number 0 cleft. J Craniofac Surg 2008;19: 1348-52.

7. Morice A, Galliani E, Amiel J, et al. Diagnostic criteria in Pai syndrome: results of a case series and a literature review. Int J Oral Maxillofac Surg 2019;48:283-90.

8. Mishima K, Mori Y, Minami K, et al. A case of Pai syndrome. Plast Reconstr Surg 1999;103:166-70.

9. Fisher DM. Unilateral cleft lip repair: an anatomical subunit approximation technique. Plast Reconstr Surg 2005;116:6171.

10. Noordhoff MS. Reconstruction of vermilion in unilateral and bilateral cleft lips. Plast Reconstr Surg 1984;73:52-61.

11. Mishra S, Sabhlok S, Panda PK, et al. Management of midline facial clefts. J Maxillofac Oral Surg 2015; 14:883-90.

12. Gillies HD, Millard DR. The principles and art of plastic surgery. Boston: Little, Brown and Company; 1957.

13. Gonzalez-Ulloa M, Castillo A, Stevens E, et al. Preliminary study of the total restoration of the facial skin. Plast Reconstr Surg (1946) 1954;13:151-61.

14. Burget GC, Menick FJ. The subunit principle in nasal reconstruction. Plast Reconstr Surg 1985;76:239-47.

15. Tse R, Lien $S$. Unilateral cleft lip repair using the anatomical subunit approximation: modifications and analysis of early results in 100 consecutive cases. Plast Reconstr Surg 2015; 136:119-30. 